\title{
COMPARATIVE EVALUATION OF THE ANTIBACTERIAL ACTIVITY OF GLASS IONOMER RESTORATION INCORPORATED WITH DIFFERENT METAL OXIDES NANOPARTICLES ON ORAL STREPTOCOCCUS MUTANS
}

\author{
Naglaa Ezzeldin *, Hisham Elshishtawy ${ }^{* *}$, Ola El-Borady *** \\ and Mona Mohamed ${ }^{* * * *}$
}

\begin{abstract}
Objective: The objective of this study is to compare and evaluate the antibacterial activity of modified conventional Glass ionomer restoration (GI) with zinc oxide ( $\mathrm{ZnO}-\mathrm{NPs}$ ) and cerium oxide Nanoparticles (CeO2-NPs) on the oral Streptococcus mutans (SM).

Materials and Methods: one hundred and five disc-shaped specimens were divided into seven groups; GI, GI incorporated with three, five and seven percent ZnO-NPs and CeO2-NPs. Antibacterial activity was assessed by counting of colony forming unit (CFU) of SM after direct contact test and 96 hours biofilm evaluation. Fourier transform infrared spectroscopy (FTIR) was used to illustrate interaction between different concentrations of Nanoparticles incorporated with GI.
\end{abstract}

Results: Direct contact test showed a significant reduction of CFU of GI incorporated with seven percent ZnO-NPs in relation to GI (P value 0.007). Biofilm evaluation showed significant reduction of $\mathrm{CFU}$ of GI incorporated with three, five and seven percent $\mathrm{ZnO}-\mathrm{NPs}$ and five and seven percent CeO2-NPs (P value 0.000). FTIR showed Peaks at 1405 and $1635 \mathrm{~cm}-1$ related to symmetric and asymmetric vibrations of $\mathrm{COO}-$, those peaks were broader and red shifted in GI incorporated with $\mathrm{ZnO}-\mathrm{NPs}$ and $\mathrm{CeO}-\mathrm{NPs}$.

Conclusion: Incorporation of conventional GI with $\mathrm{ZnO}-\mathrm{NPs}$ and $\mathrm{CeO} 2-\mathrm{NPs}$ enhance its antibacterial activity against oral SM.

KEY WORDS: Biofilm, Nanoparticles, Streptococcus mutans, Zinc oxide.

\footnotetext{
* Pediatric Dentistry department, Faculty of Dentistry, October University for Modern Sciences and Arts, Cairo, Egypt.

** Microbial Genetics department, Agricultural Genetic Engineering Research Institute, Agriculture Research Center, Cairo, Egypt.

*** Nanoscience department, Institution for Nanoscience and Nanotechnology, Kafrelsheikh University, Kafrelshikh, Egypt.

**** Conservative Dentistry department, Faculty of Dentistry, October University for Modern Sciences and Arts, Cairo, Egypt.
} 


\section{INTRODUCTION}

Secondary caries is defined as a carious lesion developed at the margins of an existing restoration. It is a one of the primary causes of failure of the restorative materials ${ }^{[1,2]}$. Enamel demineralization occurs by the adhesion of microorganism to the tooth surface and restorative materials to produce dental plaque ${ }^{[3]}$. Oral Streptococcus mutans (SM) is a main causative factor of dental plaque biofilm formation. Therefore, one of the most important methods for prevention of secondary caries is applying restorative materials to prevent bacterial growth and colonization, and it should be able to reduce acid production and dental plaque accumulation ${ }^{[4,5]}$.

One of the most popular restorative dental materials is Glass ionomer restoration (GI). GI is set based on acid base reaction ${ }^{[6,7]}$. It is being used in many dental applications as restoration of primary teeth, class V restorations, cementation of crowns, restoration by sandwich technique and as restorative materials in atraumatic restorative therapy ${ }^{[8,9]}$.

GI presented a chemical adhesion to the moist tooth structure with low coefficient of thermal expansion close to the tooth structure, in addition to fluoride release which is knowing for many years to have an antibacterial action ${ }^{[10,11]}$. In spite of these advantages, the internal cracks, air voids and high porosity of GI may allow the microleakage occurrence leading to secondary caries ${ }^{[12,13]}$. Also, some studies revealed no inhibition effect of fluoride after setting reaction as the low $\mathrm{pH}$ of GI during the setting reaction allows leaching of high amount of fluoride which relatively stops after setting ${ }^{[1]}$.

In the light of the above mentioned disadvantages of GI restoration, the incorporation of Nanoparticles with antibacterial effect were introduced ${ }^{[14,15]}$. Nano dimension of the nanoparticles allow a considerable interaction with the microorganisms which increase the antibacterial action. This can prevent the secondary caries and prolong the effectiveness of the restorative material ${ }^{[1,16]}$.
For many years, zinc was used as an antibacterial agent and incorporated in many dental products [17]. Zinc oxide Nanoparticles (ZnO-NPs) is a biocompatible material, non-toxic to human cells but more toxic to the bacteria. ZnO-NPs can inhibit the growth of oral SM and plaque formation ${ }^{[5,18,19]}$. Also, the antibacterial effect of $\mathrm{ZnO}-\mathrm{NPs}$ increase by increase of the concentration and decrease in Nanoparticles size ${ }^{[13,20]}$.

Among the metal oxides, the Nano particulate cerium oxide (CeO2-NPs) which belongs to lanthanide series and is a rare earth metal oxide, with antibacterial properties against many types of bacteria $^{[21,22]}$.

The aim of this study is to compare and evaluate the antibacterial activity of modified conventional GI restoration with $\mathrm{ZnO}-\mathrm{NPs}$ and $\mathrm{CeO} 2-\mathrm{NPs}$ on the $\mathrm{SM}$. The null hypothesis to be tested that there is no antibacterial effect of modified GI with $\mathrm{ZnO}$-NPs and $\mathrm{CeO} 2-\mathrm{NPs}$ on the SM.

\section{MATERIALS AND METHODS}

Conventional Glass ionomer (GC Gold Label 9, Tokyo, Japan) powder and liquid was modified by addition of different concentration of $\mathrm{ZnO}$-NPs and $\mathrm{CeO}_{2}$-NPs.

\section{Preparation of Nano particles:}

\section{Zinc oxide Nanoparticles ( $\mathrm{ZnO}-\mathrm{NPs})$ :}

A zinc acetate salt (Alfa Chemika, Mumbai, India) was dissolved in methanol and stirred until the entire amount was completely dissolved, then sodium hydroxide solution ( $\mathrm{pH}$ 10) (Loba Chemie PVT.LTD, Mumbai, India) was added drop by drop. A white precipitate was formed indicating the formation of $\mathrm{ZnO}-\mathrm{NPs}$. The precipitate was washed several times using distilled water, then dried at $100{ }^{\circ} \mathrm{C}^{[23]}$. 


\section{Cerium oxide Nanoparticles ( $\left.\mathrm{CeO}_{2}-\mathrm{NPs}\right)$ :}

Cerium IV oxide, nanoparticle size is $<25$ (Sigma Aldrich Company, St. Louis, USA)

\section{Characterization of $\mathrm{ZnO}$-NPs:}

The morphology of ZnO-NPs was observed by transmission electron microscopy (HR-TEM, JOEL JEM-2010, Peabody, MA, USA) operating at an accelerating voltage of $200 \mathrm{kV}$, with Gatan digital camera (Erlangshen ES500, Abingdon, UK).

\section{Preparation of tested specimens:}

One hundred and five disc-shaped specimens were prepared by using four mm diameter and two $\mathrm{ml}$ thickness Teflon mold. The specimens were divided into seven groups, control group (G1) GI without modifications. G2, G3 and G4, GI modified by 3, 5 and $7 \%$ ZnO-NPs, G5, G6 and G7, GI modified by 3,5 and $7 \% \mathrm{CeO} 2-\mathrm{NPs}$, respectively.

The different concentrations of the Nanoparticles were weighed by using electronic balance with four digits precision (Sartorius AG, Goettingen, Germany). The weighed nanoparticles were freshly mixed with GI powder by using plastic spatula. The recommended powder/liquid ratio was 3.6/1.

GI was mixed according to the manufacturer instructions and placed in the Teflon mold. The specimens were covered with Mylar strip and glass plate were pressed over the strip to squeeze the excess material and to obtain smooth and standardized surface. Specimens were left to dry for 20 minute at room temperature, then removed from the mold and sterilized by UV light for 30 minutes.

\section{Antibacterial tests:}

\section{Bacterial strain and growth condition:}

Streptococcus mutans (SM) ATCC 25175 were cultured aerobically in brain heart infusion (BHI) (Lab M, Lancashire, UK) for 24 hour at 37 ${ }^{\circ} \mathrm{C}$. The bacterial suspension prepared at optical density of $\mathrm{DO}_{600}=(0.6-0.7)$ measured by using Spectrophotometer (TECAN Nanoquant Infinite 200 Pro, Männedorf, Switzerland)

\section{Agar diffusion test:}

$200 \mu 1$ of SM suspension was spread on BHI agar plates and left for 30 minute at room temperature. Thirty five set discs (five discs from each group) were placed in direct contact with the BHI agar. The plates were incubated aerobically for 24 hours at $37^{\circ} \mathrm{C}$. The inhibition zones were measured in millimeters ${ }^{[1]}$.

\section{Direct contact test:}

Thirty five set discs were placed in 96-well microtitre plates (Cellstar, Greiner, Germany). 10 $\mu 1$ of SM suspension was spread on the surface of the discs. Five empty wells were considered as a positive control and (G1) were considered as a negative control. The plates were incubated for one hour to evaporate the liquid leaving a thin layer of bacteria in contact with the discs. Then $500 \mu \mathrm{l}$ of BHI broth were added to each well. After 24 hour a $10 \mu 1$ of the mixture (bacterial suspension + BHI broth) were diluted to $10^{-6}$, then spread on BHI agar plates and incubated aerobically for 24 hours at $37^{\circ} \mathrm{C}$. The SM colonies were counted to determine the colony forming unit $(\mathrm{CFU} / \mathrm{ml})^{[16]}$.

\section{Evaluation of biofilm:}

Thirty five set discs were placed in 96-well microtitre plates. One $\mathrm{ml}$ of bacterial suspension were added to each well, then the plates were incubated for $96 \mathrm{~h}$ at $37^{\circ} \mathrm{C}$.After 48 hours the growth media (BHI+1\% sucrose) were renewed. After incubation the discs were drained and transferred to sterile tube with $1 \mathrm{ml} \mathrm{BHI}$. The biofilm attached to the disc surface was collected by using a vortex mixer (Stuart, Staffordshire, UK) for one min at $2500 \mathrm{rpm}$. The collected bacteria was serially diluted to $10^{-6}$ and inoculated to BHI agar plates and incubated for $24 \mathrm{~h}$ at $37^{\circ} \mathrm{C}$. Finally the SM colonies were counted to determine $(\mathrm{CFU} / \mathrm{ml})^{[15]}$. 


\section{Fourier transform infrared spectroscopy (FTIR):}

FTIR spectrometer (IR Affinity-1S, Shimadzu Co, Kyoto, Japan) was used within scanning range from $400-4000 \mathrm{~cm}^{-1}$ using $\mathrm{KBr}$ as reference to demonstrate the structural composition and illustrate interaction between different concentrations of $\mathrm{ZnO}-\mathrm{NPs}$ and $\mathrm{CeO}_{2}-\mathrm{NPs}$ incorporated with GI.

\section{Statistical analysis:}

Data were statistically described in terms of mean \pm , standard deviation ( \pm SD), and range. Comparison between the study groups was done using one way analysis of variance (ANOVA) test with Bonferroni Post Hoc multiple two group comparisons. P values less than 0.05 was considered statistically significant. All statistical calculations were done using computer program IBM SPSS (Statistical Package for the Social Science; IBM Corp, Armonk, NY, USA) release 22 for Microsoft Windows.

\section{RESULTS}

\section{Characterization of $\mathrm{ZnO}$-NPs}

TEM image of ZnO-NPs was shown with a mean diameter about $20 \mathrm{~nm}$ in a uniform hollow spheres. (Figure 1)

\section{Antibacterial tests}

\section{Agar diffusion test:}

There was no inhibition zone around the specimens of different groups.

\section{Direct contact test $(D C T)$ :}

The results of CFU of SM regarding to the DCT are represented in figure 2. G3, G4 and G7 showed a significant $\mathrm{CFU}$ reduction in relation to the positive control (P value 0.000, 0.013, 0.037) respectively, while only $\mathrm{G} 4$ showed a significant $\mathrm{CFU}$ reduction in relation to $\mathrm{G} 1$ (P value 0.007).

\section{Evaluation of biofilm:}

CFU of SM after 96h biofilm formation are depicted in figure 3. All tested groups showed significant reduction of SM CFU in relation to $\mathrm{G} 1$ (P value 0.000) except G5.

\section{Fourier transform infrared spectroscopy (FTIR):}

The characteristic bands observed in all FTIR charts are tabulated in table (1), whoever. The FTIR of ZnO-NPs, G1, G2, G3 and G4 were presented in figure 4. Figure 5 showed FTIR of the $\mathrm{CeO}_{2}-\mathrm{NPs}$, G1, G5, G6 and G7.

TABLE (1) FTIR spectral bands assignment of ZnO-NPs, CeO2-NPs, GI and GI incorporated with diffrent concentrations of $\mathrm{ZnO}-\mathrm{NPs}$ and $\mathrm{CeO} 2-\mathrm{NPs}$

\begin{tabular}{|l|l|l|l|l|l|l|l|l|l|}
\hline Peak assignment & $\mathrm{G} 1$ & $\mathrm{ZnO}-\mathrm{NPs}$ & $\mathrm{CeO}_{2}-\mathrm{NPs}$ & $\mathrm{G} 2$ & $\mathrm{G} 3$ & $\mathrm{G} 4$ & $\mathrm{G} 5$ & $\mathrm{G6}$ & $\mathrm{G} 7$ \\
\hline${ }^{\circ} \mathrm{O}-\mathrm{H}^{*}$ & 3460 & 3433 & 3454 & 3430 & 3437 & 3425 & 3454 & 3443 & 2448 \\
\hline $\mathrm{Zn}-\mathrm{O}$ & ----- & 562 & ---- & 451 & 431 & 429 & ----- & ----- & ----- \\
\hline${ }^{\circ} \mathrm{C}-\mathrm{H}^{*}$ & 2363 & ----- & 2314 & 2372 & 2372 & 2372 & 2371 & 2365 & 2355 \\
\hline Symmetric COO- & 1405 & ----- & & 1420 & 1420 & 1420 & & & \\
\hline Asymmetric COO- & 1635 & ----- & 1635 & 1623 & 1623 & 1623 & 1635 & 1635 & 1635 \\
\hline Ce-O & ----- & ---- & 474 & ----- & ----- & ----- & ----- & ---- & ---- \\
\hline
\end{tabular}

${ }^{*}: v=$ stretching 


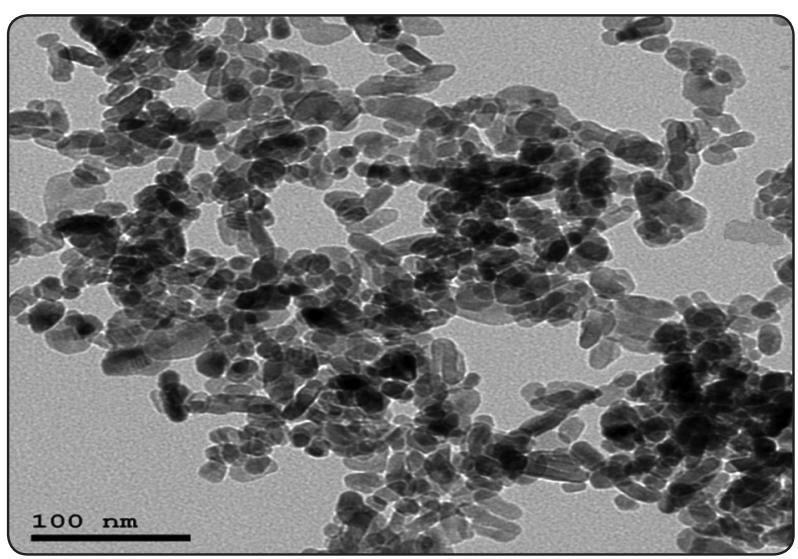

Fig. (1): TEM image represent the morphology of zinc oxide Nanoparticles

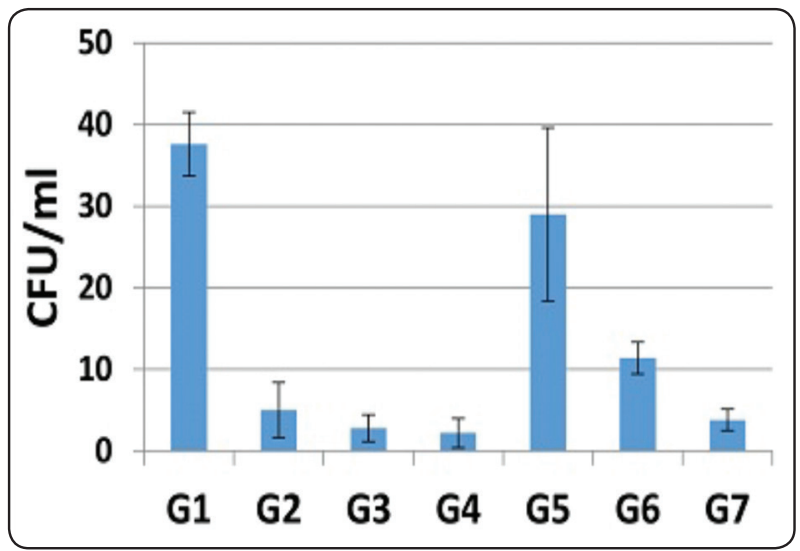

Fig. (3): Mean and SD of colony forming unit counting of Streptococcus mutans after 96 hours biofilm formation

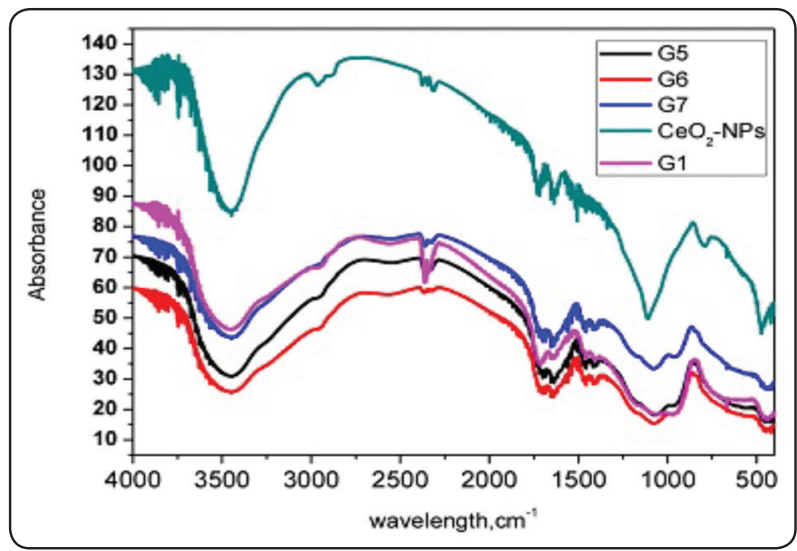

Fig. (5): FTIR spectra of the CeO2-NPs, GI and GI incorporated with different concentrations of $\mathrm{CeO} 2-\mathrm{NPs}$

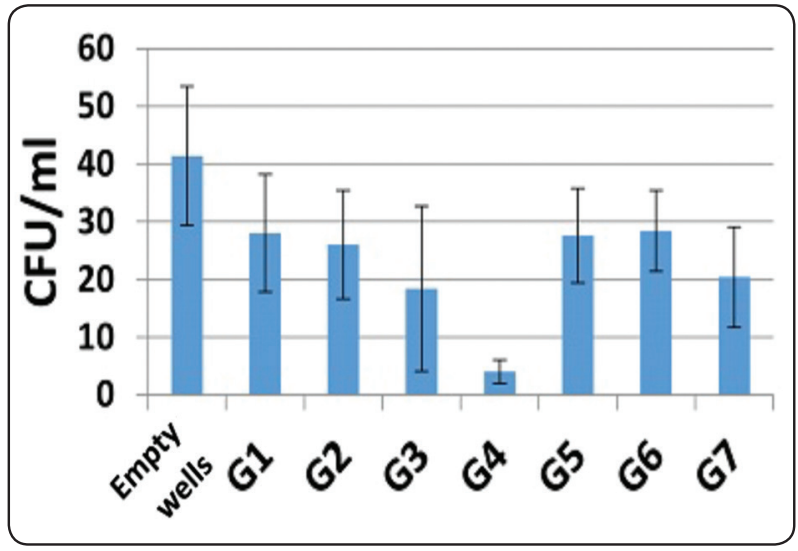

Fig. (2): Mean and SD of colony forming unit of Streptococcus mutans after direct contact test

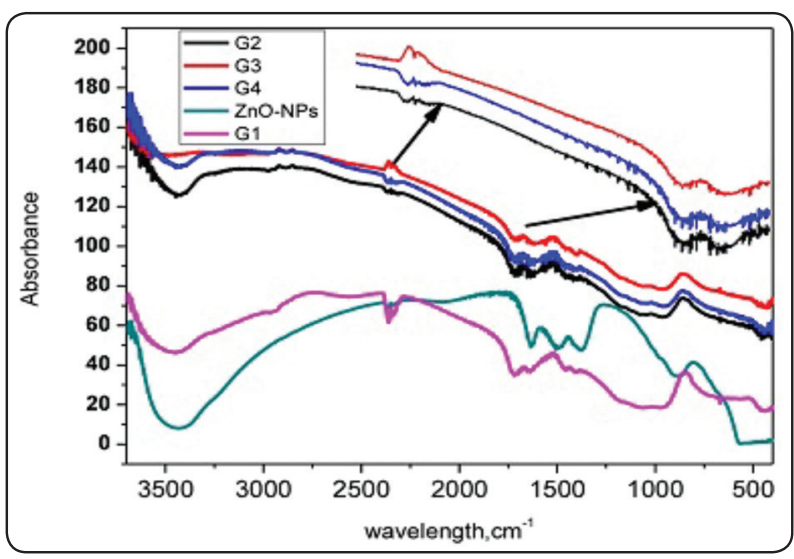

Fig. (4): FTIR spectra of the ZnO-NPs, GI and GI incorporated with different concentrations of ZnO-NPs

\section{DISCUSSION}

In the present study, the agar diffusion test didn't show any inhibition zone around all specimens. These findings were in agreement with Hojati et al. and Sungurtekin-ekci et al. ${ }^{[1,24]}$. The possible explanation for this result is the insolubility of high viscous conventional GI, ZnO-NPs and CeO2-NPs as they couldn't leash to the surrounding area to initiate an antibacterial action. The agar diffusion test is considered a traditional method to evaluate a soluble materials as antibiotics ${ }^{[24-26]}$.

However one of the most important requirement of dental restoration is a low solubility in oral 
cavity. DCT is considered a suitable test for evaluating materials with low solubility, as in DCT the bacteria was contact directly on the surface of tested materials ${ }^{[1]}$. DCT revealed that there was no significant antibacterial activity of unmodified GI in relation to the empty wells. This result was consistent with Elsaka et al. ${ }^{[27]}$. The possible explanation of this finding is that the manually mixed GI release less fluoride than the encapsulated GI due to the fact that the trituration of encapsulated GI enhances the reaction between glass particles and the cement liquid. This reaction increases the volume fraction of the GI matrix and decreases the unreacted particles. Fluoride tends to be released from the cement matrix, therefore the mechanically triturated GI would increase the fluoride release ${ }^{[28]}$. Moreover, studies revealed that the low $\mathrm{pH}$ of the freshly mixed GI could provide antibacterial, activity more than the set GI ${ }^{[29]}$.

Also, there was a significant antibacterial activity of GI incorporated with 5 and $7 \%$ concentrations of $\mathrm{ZnO}-\mathrm{NPs}$ in comparison to the empty wells and GI incorporated with 7\% $\mathrm{ZnO}-\mathrm{NPs}$ in relation to GI. This result is in agreement with Hojati et al. as they revealed that by increasing the Nanoparticles concentration the antibacterial activity increase ${ }^{[1]}$.

The mechanism of antibacterial action of $\mathrm{ZnO}-$ NPs is releasing an active oxygen species like $\mathrm{H} 2 \mathrm{O} 2$ and interacting with the bacteria with electrostatic forces, leading to the alteration of the bacterial cell wall and loss of extracellular content and bacterial cell death ${ }^{[15]}$.

GI incorporated with 7\% CeO2-NPs showed a significant antibacterial activity in relation to the empty wells but was non-significant in relation to the GI. The antibacterial action of $\mathrm{CeO} 2-\mathrm{NPs}$ has a wide range effect, not inhibiting bacterial growth but it interferes with the mitochondrial respiration function, DNA replication and cell division leading to the change of the oxidative stress induced by reactive oxygen species (ROS) and eventually cell death ${ }^{[30]}$.
To our knowledge, there is a limited number of studies that have been evaluated the antibacterial activity of $\mathrm{CeO} 2-\mathrm{NPs}$ in dental field. Christiano et al. revealed that $\mathrm{CeO} 2-\mathrm{NPs}$ showed an antibacterial effect on SM when incorporated in 10\% concentration with dental composite ${ }^{[31]}$. Also, CeO2-NPs revealed less antibacterial activity against gram positive bacteria as SM, which difficult to be penetrated with CeO2-NPs ${ }^{[22]}$. This fact could explain the lower antibacterial activity of $\mathrm{CeO} 2-\mathrm{NPs}$ in relation to $\mathrm{ZnO}-\mathrm{NPs}$, which have a higher antibacterial effect against gram positive bacteria ${ }^{[32,33]}$.

In addition to the role of Nanoparticles concentration in antibacterial activity against SM. The present study revealed that the antibacterial activity of the GI incorporated with ZnO-NPs and CeO2-NPs have been enhanced after $96 \mathrm{~h}$ biofilm maturation, these results comply with Hojati et al. ${ }^{[1]}$, who revealed that the antibacterial activity of the dental restorations incorporated with Nanoparticles increased by time.

The role of Zinc oxide in biofilm inhibition could be explained by its ability to inhibit sugar transportation and metabolism. As well as distribution of enzymes systems of dental biofilms by displacing magnesium ions which are essential for enzymatic activity of the dental plaque. Also, Zinc ions is able to reduce acid production by inhibition of glucosyltransferase activity ${ }^{[11]}$.

The null hypnosis of this study was rejected, as the incorporation of conventional GI with ZnO-NPs and $\mathrm{CeO} 2-\mathrm{NPs}$ enhances its antibacterial activity against oral SM.

In the present study, the functional groups of ZnONPs, CeO2-NPs, unmodified GI and GI incorporated with various concentrations of Nanoparticles were measured using FTIR. ZnONPs FTIR spectrum was established a broad peak around $3433 \mathrm{~cm}^{-1}$ which was expressed to the $\mathrm{O}-\mathrm{H}$ stretching mode of hydroxyl group of residual organic species or adsorbed water persisted during ZnONPs 
preparation. This peak showed in unmodified GI at $3460 \mathrm{~cm}^{-1}$, and at 3430,3437 and $3425 \mathrm{~cm}^{-1}$ after GI incorporation with 3, 5, 7\% ZnONPs, respectively. Moreover, ZnONPs revealed a $\mathrm{Zn}-\mathrm{O}$ peak at 562 $\mathrm{cm}^{-1}$, which was in accordance to the previously obtained by Sowribabu et al ${ }^{[34]}$. This peak has been red shifted after GI modification with ZnONPs and appeared at 451,431 and $429 \mathrm{~cm}^{-1}$ for 3, 5, and 7\%, respectively.

The FTIR spectrum of unmodified GI showed two fork like small peaks centered at $2363 \mathrm{~cm}^{-1}$ attributed to $\mathrm{C}-\mathrm{H}$ vibration mode. These peaks were appeared at $2372 \mathrm{~cm}^{-1}$ after incorporation with ZnONPs. Also, GI was showed emerged peaks at 1405 and $1635 \mathrm{~cm}^{-1}$ related to symmetric and asymmetric tensile vibrations of COO- (in carboxylic acid salt compounds) ${ }^{[35]}$. These peaks were looked broader and shifted to 1420 and $1623 \mathrm{~cm}^{-1}$ after GI modification with $\mathrm{ZnONPs}$. It may be attributed to the incorporation of $\mathrm{ZnO}$ to the active - $\mathrm{COO}$ group in GI. This suggestion is confirmed by the decrease in the intensity observed in the $\mathrm{C}=\mathrm{O}$ stretch of polyacrylic acid at $1720 \mathrm{~cm}^{-1}$, which could be explained by the formation of polyacrylate salts ${ }^{[36]}$.

Incorporation of GI with $\mathrm{CeO}_{2}$-NPs influnced the absorption of some peaks. For example, the peak located around $1720 \mathrm{~cm}^{-1}$ in unmodified GI which was corresponding to $\mathrm{C}=\mathrm{O}$ stretch of polyacrylic acid showed a small decreas in its intensity. On the other hand, the peak at $1635 \mathrm{~cm}^{-1}$ assigned to asymmetric tensile vibrations of COO- seemed sharper. However, peaks in the region from 2300$600 \mathrm{~cm}^{-1}$ attributed to organic species did not show any change.

In FTIR spectrum of $\mathrm{CeO}_{2}$-NPs, the peak was developed at $474 \mathrm{~cm}^{-1}$ which could be explained by the presence of metallic $\mathrm{Ce}$ ion [37]. This peak was disappeared after incorporation of GI with $\mathrm{CeO}_{2}$ NPs, which was indicated the linkage between $\mathrm{CeO}_{2}$-NPs and GI. These results are consistent with that obtained by Sowribabu et al. ${ }^{[38]}$.

\section{CONCLUSION}

Incorporation of conventional GI with $\mathrm{ZnO}-\mathrm{NPs}$ and $\mathrm{CeO} 2-\mathrm{NPs}$ enhances its antibacterial activity against oral SM. The FTIR confirmed that, the interaction between the Nanoparticles and GI was occurred through the COO- functional group of polyacrylic acid in GI.

\section{REFERENCES}

1. Hojati ST, Alaghemand H, Hamze F, et al. Antibacterial, physical and mechanical properties of flowable resin composites containing zinc oxide nanoparticles. Dent Mater 2013;29:495-505.

2. Deepalakshmi M, Poorni S, Miglani R, et al. Evaluation of the antibacterial and physical properties of glass ionomer cements containing chlorhexidine and cetrimide: an invitro study. Indian J Dent Res 2010;21:552-6.

3. Ahn SJ, Lee SJ, Kook JK, et al. Experimental antimicrobial orthodontic adhesives using nanofillers and silver nanoparticles. Dent Mater 2009;25:206-13.

4. Simon-Soro A, Mira A. Solving the etiology of dental caries. Trends Microbiol 2015;23:76-82.

5. Hafshejani TM, Zamanian A, Venugopal JR, et al. Antibacterial glass-ionomer cement restorative materials : A critical review on the current status of extended release formulations. J Control Release 2017;262:317-28.

6. Nicholson J. Adhesion of glass-ionomer cements to teeth: A review. Int J Adhes Adhes 2016;69:33-8.

7. Kim DA, Lee JH, Jun SK, et al. Sol - gel-derived bioactive glass nanoparticle-incorporated glass ionomer cement with or without chitosan for enhanced mechanical and biomineralization properties. Dent Mater 2017;33:805-17.

8. Najeeb S, Khurshid Z, Zafar MS, et al. Modifications in glass ionomer cements : nano-sized fillers and bioactive nanoceramics. Int J Mol Sci 2016;17:1-14.

9. deAmorimRG,Leal SC,Frencken JE. Survival of atraumatic restorative treatment (ART) sealants and restorations : A meta-analysis. Clin Oral Investig 2012;16:429-41

10. Chau NPT, Pandit S, Cai JN, et al. Relationship between fluoride release rate and anti-cariogenic biofilm activity of glass ionomer cements. Dent Mater 2015;31:100-8.

11. Vanajassun P. Effects of zinc oxide nanoparticles in combination with conventional glass ionomer cement: In vitro study. Adv Hum Biol 2014;4:31-6 
12. Ilie N, Hickel R, Valceanu AS, et al. Fracture toughness of dental restorative materials. Clin Oral Investig 2012;16:489-98.

13. Agarwal P, Nayak R, Upadhya PN, et al. Evaluation of properties of Glass Ionomer cement reinforced with Zinc Oxide nanoparticles - An in vitro study. Mater Today Proc 2018;5:16065-72.

14. Thorat SB, Diaspro A, Salerno M. In vitro investigation of coupling-agent-free dental restorative composite based on nano-porous alumina fillers. J Dent 2014;42:279-86.

15. Petromilli Nordi Sasso Garcia P, Cardia M, Francisconi $\mathrm{R}$, et al. Antibacterial activity of glass ionomer cement modified by zinc oxide nanoparticles. Microsc Res Tech 2017;80:456-61.

16. Kasraei S, Sami L, Alikhani M, et al. Antibacterial properties of composite resins incorporating silver and zinc oxide nanoparticles on Streptococcus mutans and Lactobacillus. Restor Dent Endod 2014;39:109-14.

17. Almoudi MM, Hussein AS, Abu Hassan MI, et al. A systematic review on antibacterial activity of zinc against Streptococcus mutans. Saudi Dent J 2018;30:283-91 .

18. Sirelkhatim A, Mahmud S, Seeni A, et al. Review on zinc oxide nanoparticles: antibacterial activity and toxicity mechanism. Nano-micro lett 2015;7:219-42.

19. Liu W, Su P, Chen S, et al. Synthesis of TiO 2 nanotubes with $\mathrm{ZnO}$ nanoparticles to achieve antibacterial properties and stem cell compatibility. Nanoscale 2014;6:9050-62.

20. Dizaj SMF, Lotfipour F, Barzegar-Jalali $M$, et al. Antimicrobial activity of the metals and metal oxide nanoparticles. J Mater Sci Eng 2014;44:278-84.

21. Kannan S, Sundrarajan M. A green approach for the synthesis of a cerium oxide nanoparticle: characterization and antibacterial activity. Int J Nanosci 2014;13:1450018.

22. Passos Farias I, Santos C, Sampaio F. Antimicrobial activity of cerium oxide nanoparticles on opportunistic microorganisms : A systematic review. Biomed Res Int 2018; 2018:1-14.

23. Dutta S, Ganguly BN. Characterization of $\mathrm{ZnO}$ nanoparticles grown in presence of Folic acid template. $\mathrm{J}$ Nanobiotechnol 2012;10:1-10.

24. Sungurtekin-ekci E, Ozdemir-ozenen D, Duman S, et al. Antibacterial surface properties of various fluoridereleasing restorative materials in vitro. J Appl Biomater Funct Mater 2015;13:169-73.

25. Aydin Sevinc B, Hanley L. Antibacterial activity of dental composites containing zinc oxide nanoparticles. J Biomed Mater Res B Appl Biomater 2010;94:22-31.
26. Dahle JT, Arai Y. Environmental geochemistry of cerium : applications and toxicology of cerium oxide nanoparticles. Int J Environ Res Public Health 2015;12:1253-78.

27. Elsaka SE, Hamouda IM, Swain MV. Titanium dioxide nanoparticles addition to a conventional glass-ionomer restorative: Influence on physical and antibacterial properties. J Dent 2011;39:589-98.

28. Hammouda IM. Zinc interaction with glass-ionomer restorative materials. J Rehabilit Robotics 2013;1:54-70.

29. Tuzuner T, Kusgoz A, Er K, et al. Antibacterial activity and physical properties of conventional glass-ionomer cements containing chlorhexidine diacetate/cetrimide mixtures. J Esthet Restor Dent 2011;23:46-55.

30. Munusamy S, Bhakyaraj K, Vijayalakshmi L, et al. Synthesis and characterization of cerium oxide nanoparticles using curvularia lunata and their antibacterial properties. Int J Innov Res Sci Eng Technol 2014; ISSN (Online): 2347-3207.

31. Dos Santos CCL, Passos Farias IA, Reis Albuquerque AdJd et al. Antimicrobial activity of nano cerium oxide (IV) (CeO2) against Streptococcus mutans. BMC Proc 2014;8:48

32. Azam A, Ahmed AS, Oves M, et al. Antimicrobial activity of metal oxide nanoparticles against Gram-positive and Gram-negative bacteria: A comparative study. Int $\mathrm{J}$ Nanomedicine 2012;7:6003-9.

33. Emami-karvani Z, Chehrazi P. Antibacterial activity of $\mathrm{ZnO}$ nanoparticle on gram- positive and gram-negative bacteria. Afr J Microbiol Res 2011;5:1368-73.

34. Sowribabu K, Reddy A, Cherukuru S, et al. Synthesis and optical characterization of porous $\mathrm{ZnO}$. J Adv Ceram 2013;2:260-65.

35. Nourmohammadi J, Sadrnezhaad S, Behnamghader A. In vitro bioactivity of novel cured ionomer cement based on iron oxide. Ceram Int 2010;36:1645-51.

36. Aguiar H, Serra J, González P, et al. Structural study of sol-gel silicate glasses by IR and Raman spectroscopies. J Non Cryst Solids 2009;355:475-80.

37. El-Damrawi G, Behairy A, Abdelghany AM, Structural characterization of novel cerium phosphate glass ionomer cements (GICs) doped with $\mathrm{GaCl}$ (Phthalocyanine). New J Glass Ceram 2018;8:23-38.

38. Sowribabu K, Reddy A, Cherukuru S, et al. Glass-ionomer cement $\mathrm{SiO} 2, \mathrm{~A} 12 \mathrm{O} 3, \mathrm{Na} 2 \mathrm{O}, \mathrm{CaO}, \mathrm{P} 2 \mathrm{O} 5$, F-Containing alternative additive of $\mathrm{Zn}$ and $\mathrm{Sr}$ prepared by sol-gel method. Egypt J Biophys Biomed Engng 2012;13:53-72. 